\title{
An Ultra High speed Low power Low settling time error and wide dynamic range voltage Continuous-time Common-Mode Feedback Circuit in 0.18 $\mu \mathrm{m}$ CMOS
}

\author{
Sina MAHDAvi ${ }^{1 *}$, Arefeh Soltani ${ }^{1}$, Maryam PoreH ${ }^{1}$, Shima Tayyeb GHASEMI ${ }^{1}$, \\ and Tohid Moradi KHANSHAN ${ }^{2}$ \\ ${ }^{1}$ Department of Microelectronic Research Urmia Graduate Institute \\ Urmia, Iran \\ ${ }^{2}$ Department of Microelectronic Research Urmia university \\ Urmia, Iran \\ m.s.mahdavi@urumi.ac.ir, m.a.soltani@urumi.ac.ir, m.m.poreh@urumi.ac.ir , \\ m.s.tayyebghasemi@urumi.ac.ir, and t.moradi@urmia.ac.ir
}

\begin{abstract}
A novel method to design continuous-time common-mode feedback circuit is presented in this paper. The main purposes of the proposed idea are increasing the speed and decreasing the settling time error of the common-mode feedback circuit, besides it has a wide dynamic rang voltage to set the output in desired value as well. As MATLAB simulation result shows the proposed circuit can adjust the output in reliable value by applying the reference voltage (Vref) from 0.7 to 1.3 volt very well. Also, the mentioned structure is a proper choice for low voltage application too. The power consumption of the proposed common-mode feedback circuit is just $200 \mu \mathrm{W}$ with power supply of 1.8 volts, and $0.5 \mathrm{pF}$ capacitor load is applied at the output nodes of the amplifier. Meanwhile, the proposed circuit is simulated in whole process corner too. Simulation results are performed using

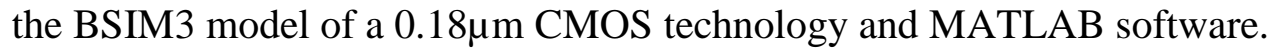

Keywords: Common-mode feedback, folded cascode, low voltage, high speed

\section{INTRODUCTION}

Fully differential op-amps have many advantages over their single-ended counterparts such as large output voltage swing for a given voltage rail, better immunity to supply and common-mode noise and lower even-order harmonics. On the other hand, doubling in most parts of the circuit and requiring additional negative feedback control circuit, referred to as the common-mode feedback circuit, are disadvantages of fully differential structures. Using fully differential op-amps in a feedback application, applied feedback determines differential signal voltages, but doesn't affect common-mode output voltage. Therefore, it is necessary to add a common-mode feedback circuit to stabilize voltage at a proper common-mode voltage that maximizes the op-amp output swing [1].

Common-mode feedback circuits (CMFB) stabilize common-mode voltages for fully differential analog systems by means of adjusting the common-mode output currents. The two differential output voltages are averaged and compared with the common-mode reference voltage (Vref), and the differential voltage is converted to the common-mode output current to adjust the common-mode voltage [2]. Various studies have suggested the use of common-mode feedback to reduce the common-mode signal but none of measured results are given to show the application of CMFB in real time application [3]. Three commonly used common-mode feedback circuits are: a passive R-C common-mode feedback circuit, switched capacitor common-mode feedback circuit and an active differential-pair common-mode feedback circuit. The R-C common-mode feedback circuit can operate at different supplies, but requires large resistance to maintain the high DC gain 
and large output swing. The switched-capacitor common-mode feedback circuit also has smaller passive area, but its loop bandwidth and stability are very sensitive to the supply voltage because of the supply-dependent on resistance of the switches. Differential-pair common-mode feedback circuit doesn't need large area passive components, but it has limited linear signal range for the main amplifier and is not suitable at low supply [4]. In this paper a novel high speed low settling time error and wide dynamic range voltage based on differential-pair continuous-time commonmode feedback circuit is presented. Also the proposed circuit is a proper choice for using low voltage applications too, because it needs just fewer voltage to start its performance.

The proposed paper is organized as follow: in section 2 different fully differential amplifiers are discussed. Proposed common-mode feedback is presented in section 3. In section 4 simulation results of the paper is given and finally section 5 concludes the paper.

\section{Different Operational Transconductance Amplifiers}

Operational Amplifiers are the basic building blocks of any analog integrated circuits such as continuous-time filters, variable gain amplifiers, analog to digital converters, etc. Designing a high gain and high speed OTA poses a challenge in deep submicron technologies as the reduction in the feature size results in the reduction of the transistor gain factor numerous analog circuits Since analog circuit performance is strongly dependent on gain and speed techniques to improve gain and speed are indispensable in deep submicron technologies [5]. OTA is basically an op-amp without an output buffer which drive a large capacitive loads. Some of its main characteristics are Gain, Unity Gain Bandwidth, Phase margin, Output swing, Slew rate, CMRR, and PSRR. Single stage fully differential op-amps are usually used to attain higher unity gain frequency owing to their fewer number of poles compared to that of the multiple stage op-amps. Since in single stage amplifiers wholly expected gain is to be achieved in one stage, cascode structure would be a choice to get higher gain. Telescopic cascode and folded cascode structures are two known instances.

In order to achieve high gain, as discussed, the differential telescopic topology can be used. The telescopic architecture is a candidate for a low power, low noise and high gain OTA [6]. The performance of simple telescopic OTA is limited by its input and output voltage swing. However, it provides high gain as well as high speed. As a result this structure is not an option for systems with low supply voltages. In order to surmount some drawbacks of telescopic operational amplifier, a folded cascode OTA can be used. Although it consumes more power compared to telescopic cascode, it provides higher output swing. Additionally, this structure sustains high gain and bandwidth as well as convenient voltage headroom for systems with low supply voltages [7]. Therefore for achieving mentioned parameters as well as in the fully differential amplifiers the common-mode feedback circuit is so necessary. The proposed common-mode feedback can apply on various fully differential amplifiers very well, but in this paper it is exerted on a folded cascode amplifier. 


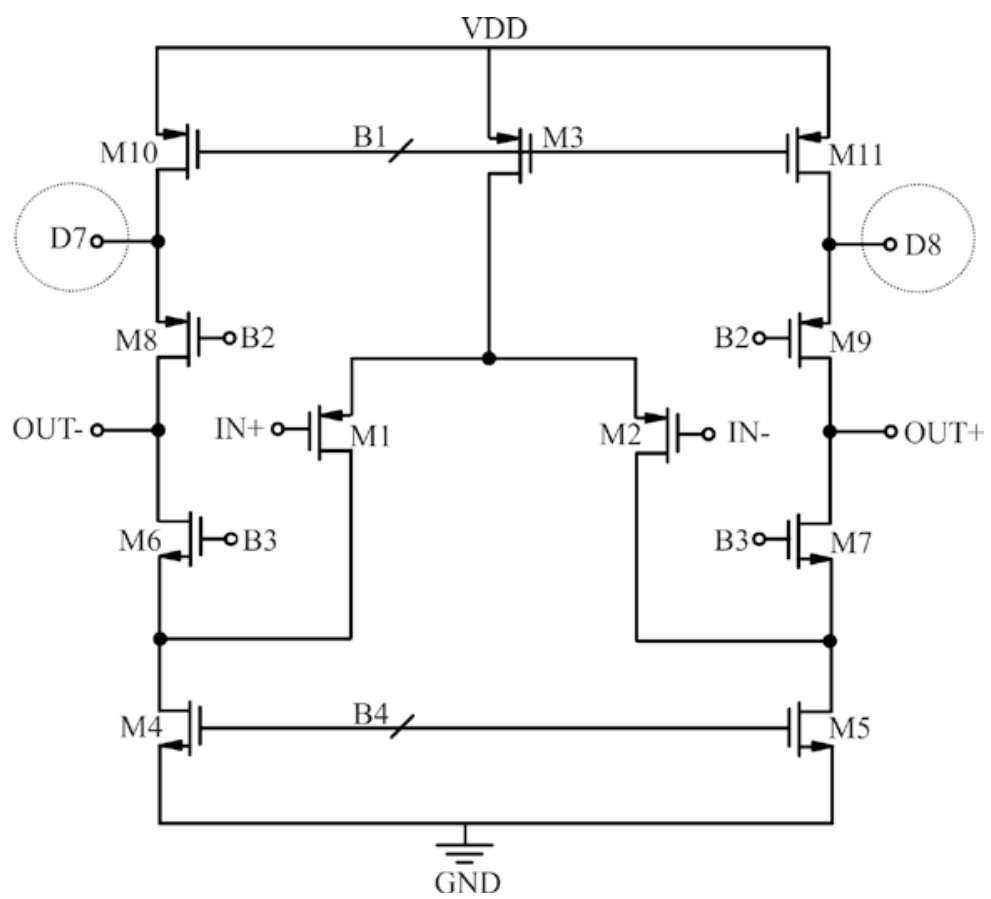

Fig.1: Folded cascode amplifier

\section{The Proposed Common-mode feedback Block (CMFB) circuit}

The proposed Common-mode feedback Block (CMFB) circuit is shown in Figure 2.

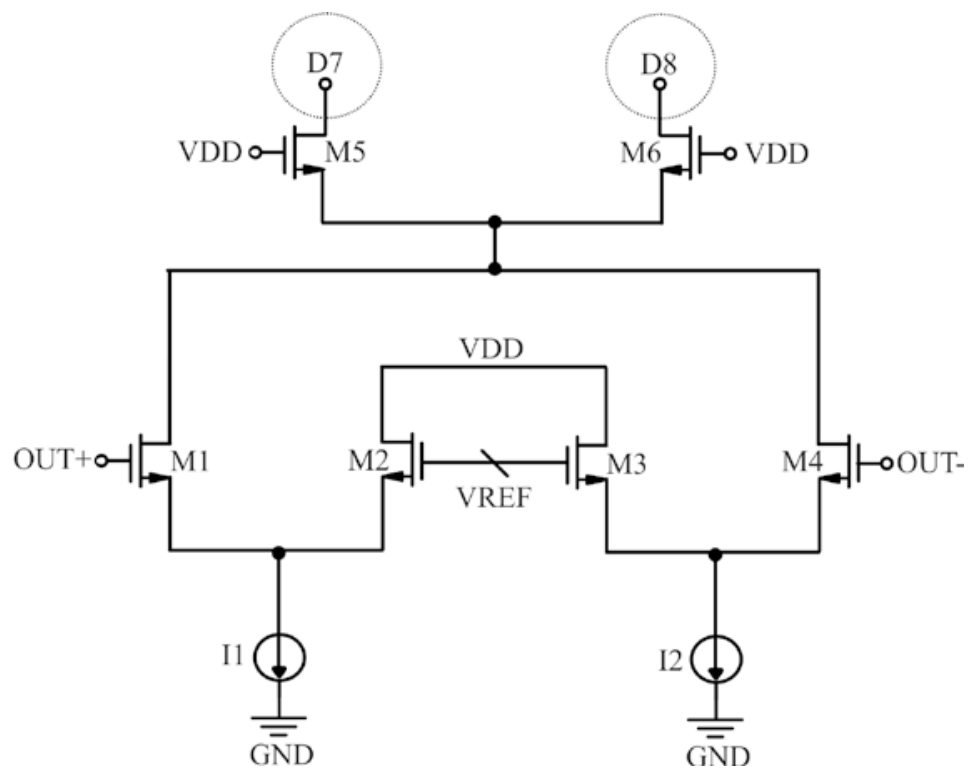

Fig. 2: The proposed common-mode feedback Block (CMFB)

In this circuit the differential-pair of M1 and M4 are sensors of the common-mode feedback in order to sense of the output voltage differences in differential type amplifiers, and the differential-pair of M5 and M6 works like an actuator to set output voltage differences in desired value. Meanwhile, the reference voltage (Vref $=\mathrm{Vdd} / 2)$ is applied on M2 and M3 to compare the output values. In the proposed CMFB circuit, the basic idea is eliminating the diode-connected transistor which applies the error correction signal to the folded cascode op-amp. The diode- 
connected transistor produces a huge delay in the feedback signal's path. By removing this transistor, the speed of the CMFB circuit will be increase extremely [1]. Therefore, the main purpose of the proposed CMFB circuit is to enhance the speed and precision of the conventional differential difference amplifier (DDA) CMFB circuit while simultaneously conserving other characteristics of the DDA circuit such as unity gain frequency, 3-dB bandwidth, phase margin and linearity. Equations (1) and (2) show the output swing voltages in conventional and proposed CMFB respectively where $\Delta \mathrm{V}$ and $\mathrm{V}$ th are overdrive voltage and threshold voltage of the transistor respectively. As equation (2) shows that by using this method, the threshold voltage of the equation (1) is removed as well. Due to this fact, the proposed idea is suitable for low voltage applications too, because it needs just fewer voltage (about 3 over drive voltage) to start its performance. Hence, the proposed CMFB circuit has high accuracy and high speed besides it can employ the low power consumption and low voltage applications too. Also, the other main feature of the mentioned CMFB circuit is working in widely range voltage with low error, it means that if Vref attuned near to 0.5 to 1.5 volt, during that range the output voltage of the main circuit can be settled in desired value. Meanwhile, for adjusting the Vref during the 0.7 to 1.3 volt the proposed CMFB circuit can work in the best condition. Due to that, the voltage of the output nodes is remained near to desired quantity as well, also all of the transistors operates in saturation region.

Output Swing $=$ Vdd $-3 \Delta V-V$ th

Output Swing $=\mathrm{Vdd}-3 \Delta \mathrm{V}$

\section{Simulation Results}

In this section simulation results of the proposed circuit are presented. Figure 3 shows the transient response of the output common-mode voltage, as it clear in mentioned figure by applying the worst case simulation (initial condition 0 and 1.8 volt) on proposed circuit, the output can settled in the desired value just after 3.7ns. The wide dynamic range voltage MATLAB simulations are shown in figure 4 and 5 . As figure 4 shows that by exerting value of reference voltage (Vref) between 0.7 to 1.3 volt the suggested common-mode voltage can keep the output voltage in desired value (low error) as well. Also figure 5 shows the same simulation by applying Vref between 0.5 and 1.5 volt. The current FFT spectrum of the common-mode feedback and the FFT of the output Folded cascode amplifier are depicted in figure 6 and 7 respectively. As simulation results show that the proposed structure has good linearity too. Finally, the Microphotograph of the proposed circuit is shown in figure8. Meanwhile, the circuit has been designed in a typical $0.18 \mu \mathrm{m}$ CMOS process with a power supply of $1.8 \mathrm{~V}$ and simulated by HSPICE software using level 49 parameters (BSIM3v3) and MATLAB software.

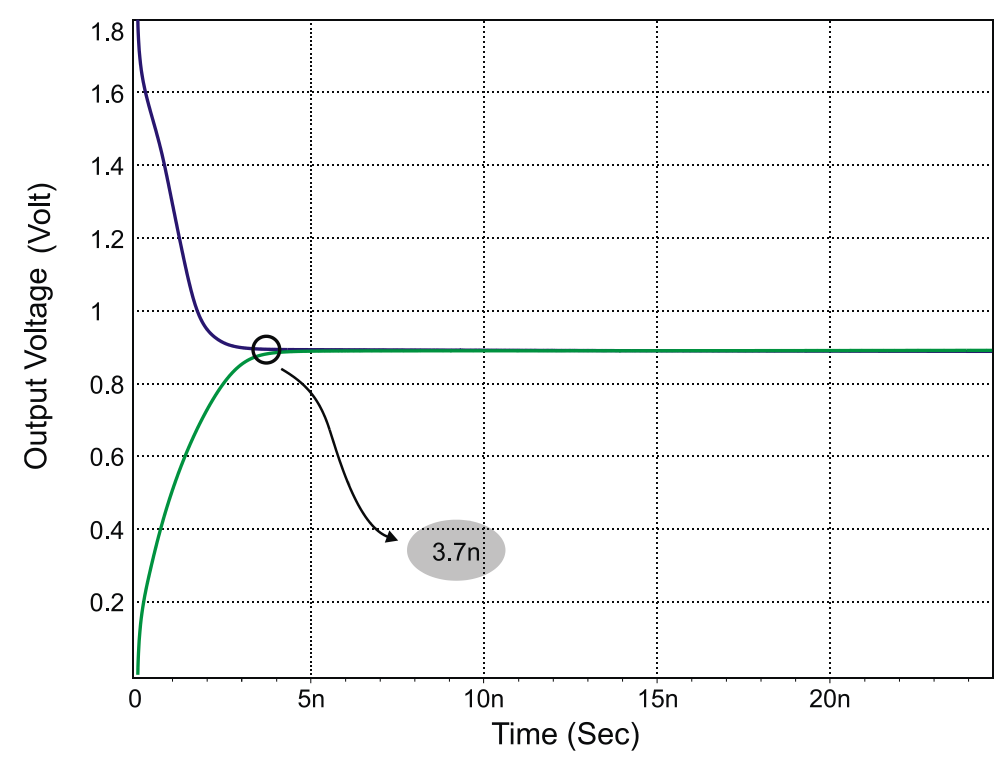

Fig. 3: Transient response of the output common-mode voltage 
Bulletin de la Société Royale des Sciences de Liège, Vol. 85, 2016, p. 1457 - 1464

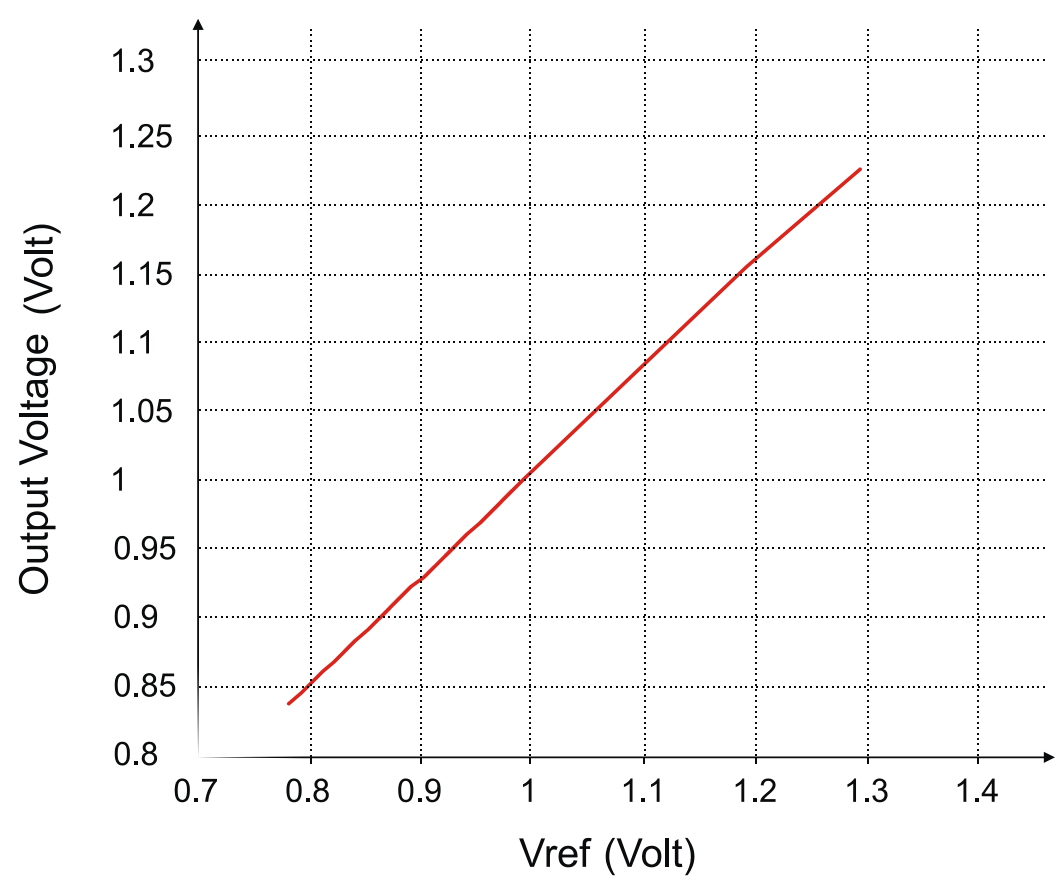

Fig. 4: Output common-mode voltage error: $0.7<$ Vref $<1.3$

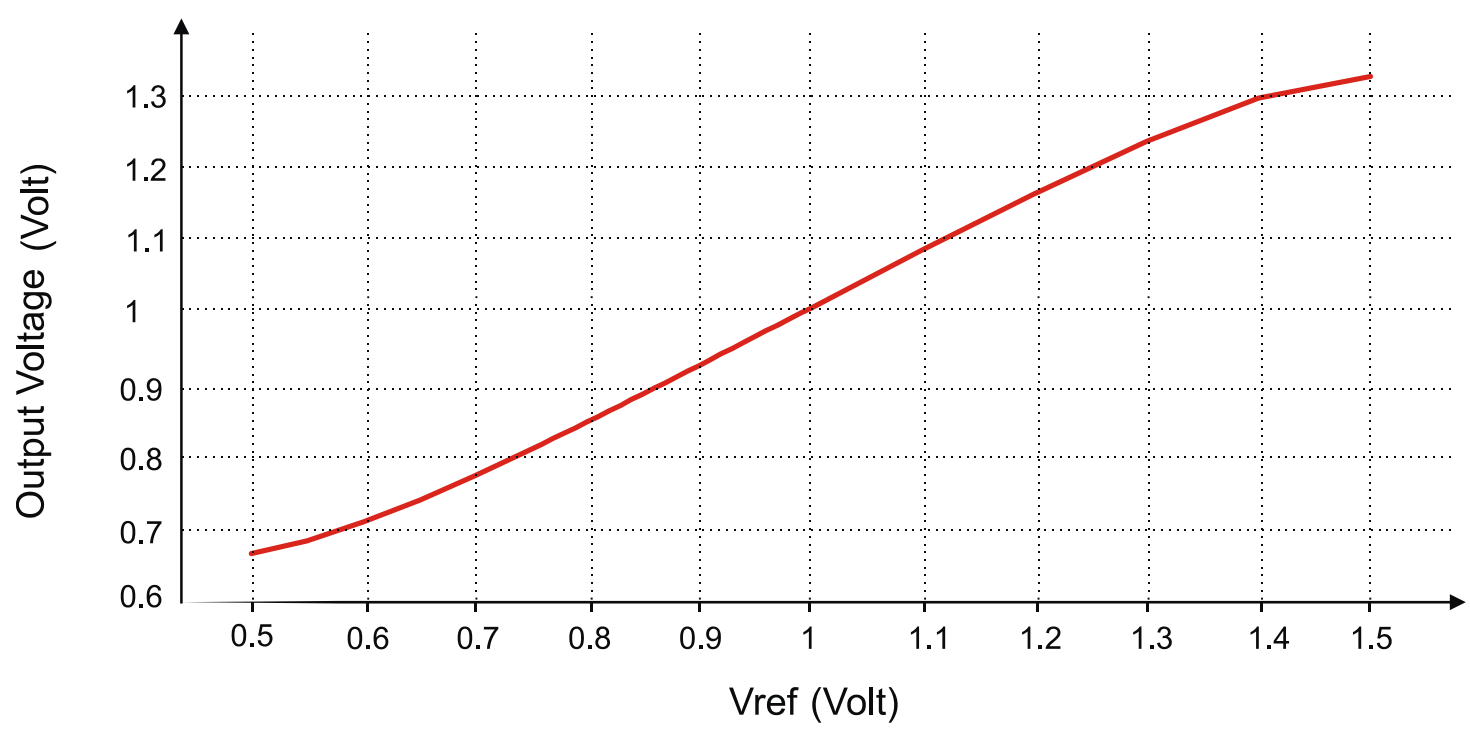

Fig. 5: Output common-mode voltage error: $0.4<$ Vref $<1.6$ 
Bulletin de la Société Royale des Sciences de Liège, Vol. 85, 2016, p. 1457 - 1464

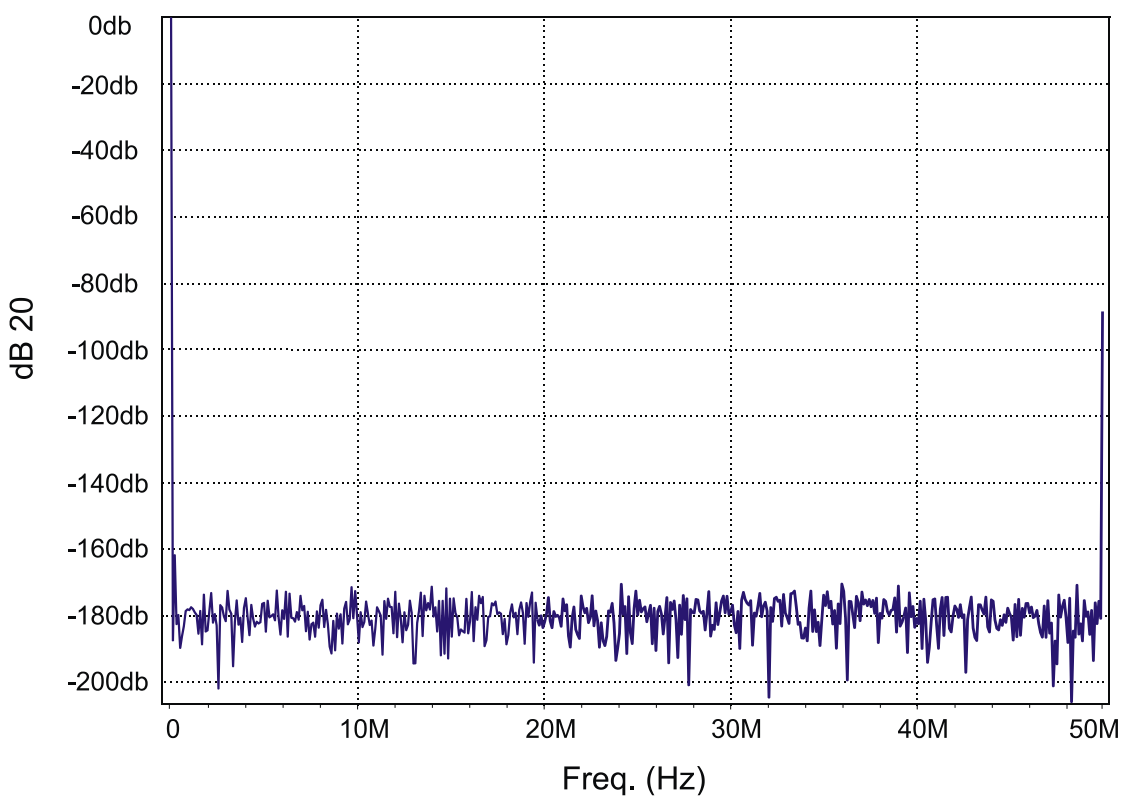

Fig. 6: Current FFT spectrum ( $\left.\mathrm{I}_{\mathrm{CMFB}}\right)$ of common-mode feedback Block (CMFB)

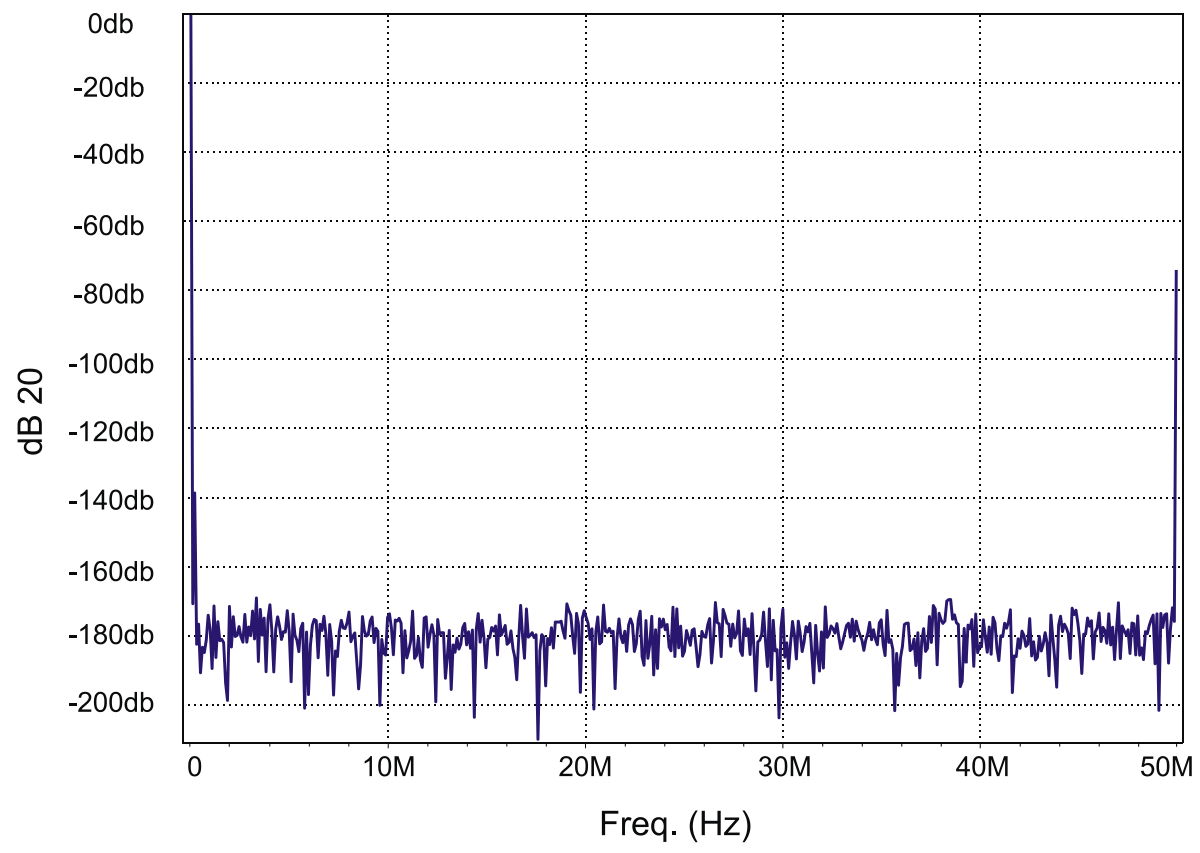

Fig. 7: Output FFT spectrum of amplifier 


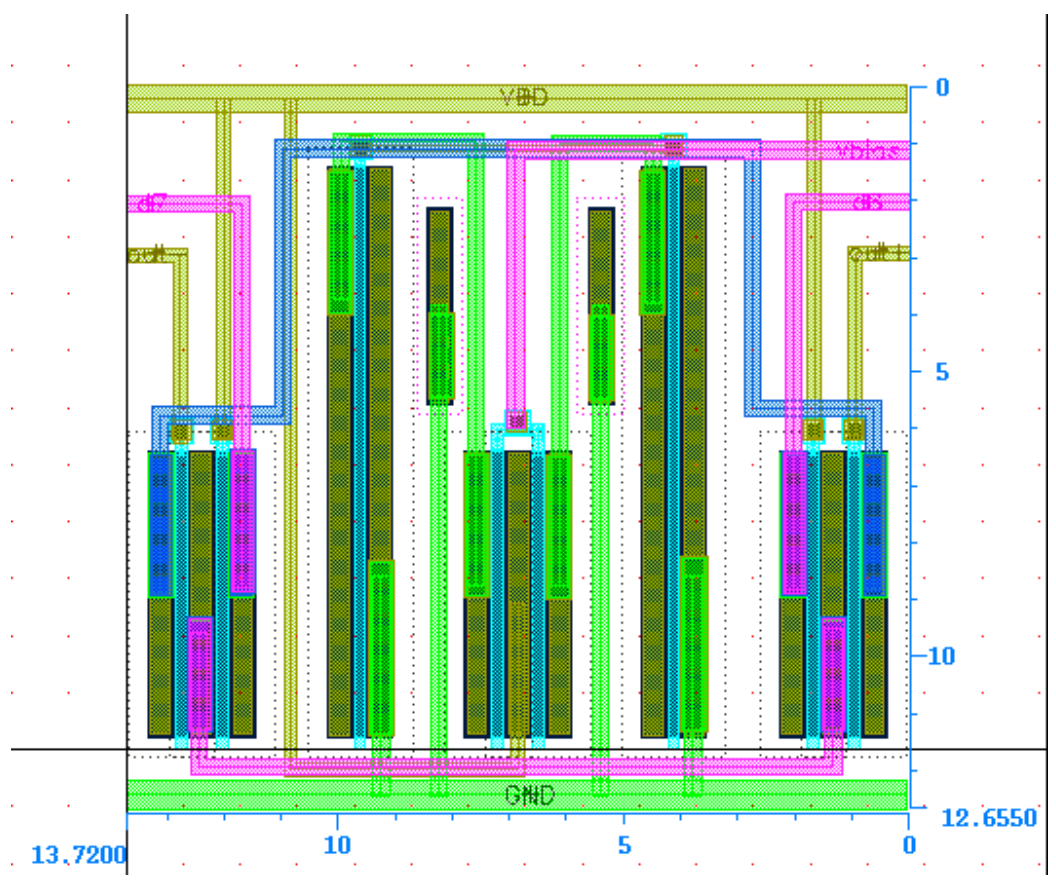

Fig. 8: Layout of the proposed CMFB

\section{Conclusion}

In this paper a novel method to design a common-mode feedback circuit based on continuous-time is presented. The main purposes of the proposed idea are increasing the speed and decreasing the settling time of the common-mode feedback circuit. Meanwhile, the suggested circuit has a wide dynamic rang voltage to set the output in desired value well. As MATLAB simulation result shows that the proposed circuit can adjust the output in reliable value by applying the Vref from 0.7 to 1.3 volt very well. Also, the mentioned structure is a proper choice for low voltage applications too, because it needs just a few voltage to starts its performance. The power consumption of the proposed circuit is just $200 \mu \mathrm{W}$ with power supply of 1.8 volt, also table 1 compare the work with previous one. Simulation results are performed using the BSIM3 model of a 0.18 $\mu \mathrm{m}$ CMOS technology and MATLAB software

Table 1: Comparison Table

[11] [1] [2] This work

\begin{tabular}{ccccc}
\hline Technology & $0.13 \mu \mathrm{m}$ & $0.35 \mu \mathrm{m}$ & $2 \mu \mathrm{m}$ & $0.18 \mu \mathrm{m}$ \\
\hline $\begin{array}{c}\text { Supply } \\
\text { Voltage }\end{array}$ & $1.8 \mathrm{~V}$ & $3.3 \mathrm{~V}$ & $5 \mathrm{~V}$ & $1.8 \mathrm{~V}$ \\
\hline $\begin{array}{c}\text { Settling } \\
\text { speed }\end{array}$ & $40 \mathrm{~ns}$ & $1.1 \mathrm{~ns}$ & $2 \mathrm{ps}$ & $3.7 \mathrm{~ns}$ \\
\hline $\begin{array}{c}\text { Settling } \\
\text { error }\end{array}$ & - & $0.35 \mathrm{mV}$ & - & $0.15 \mathrm{mV}$ \\
\hline $\begin{array}{c}\text { Power } \\
\text { consumption }\end{array}$ & $9 \mathrm{~mW}$ & - & $0.27 \mathrm{~mW}$ & $200 \mu \mathrm{W}$ \\
\hline Chip area & - & - & - & $13.72 \mu \mathrm{m}^{*} 12.65 \mu \mathrm{m}$ \\
\hline
\end{tabular}




\section{REFERENCES}

[1] T. Moradi Khaneshan, S. Naghavi, M. Nematzade, Kh. Hadidi, A.Abrishamifar, A. Khoei. “A Fast and Low Settling Error Continuous -Time Common-Mode Feedback Circuit Based On Differential Difference Amplifier” Journal of Circuits, Systems, and Computers (C) World Scientific Publishing Company. 2013

[2] L.L. John Choma, Jr. J. Draper “A Continuous-Time Common-Mode Feedback Circuit (CMFB) for High-Impedance Current Mode Application” 0-7803-5008- 1/98/ 10.0001998 IEEE. 1998

[3] M. Rahal, A. Demosthenous, R. Bayford. “An Integrated Common-Mode Feedback Topology for Multi-Frequency Bio impedance Imaging”. 978-1-4244-4353-6 /09 /\$25 .00 @2 009 IEEE.2009

[4] C. W. Hsu, P.R. Kinget "A Supply-Scalable Differential Amplifier with Pulse-Controlled Common-Mode Feedback”. 978-1-4799-3286-3/14/\$31.00 (C2014 IEEE. 2014

[5] S.Kumaravel, B.Venkataramani, S.Rishi, V.Sri Vijay, B.Shailendra "An Enhanced Folded Cascode OTA with push pull Input Stage” 978-1-4673-1591-3/12/\$31.00 C2012 IEEE. 2012

[6] S. Sharma, P. Kaur, T.Singh, M. Kumar, "A Review of Different Architectures of Operational Transconductance Amplifier,”ISSN : 2230-7190 (Online) | ISSN : 2230-9543

[7] B. Razavi , “ Design of analog integrated CMOS circuits book,”

[8] F. Castafio, G. ToreU, R. Perez-Aloe, J. M. Carrillo "Low-Voltage Rail-to-Rail Bulk-Driven CMFB Network with Improved Gain and BandWidth” 978-1-4244-8157 -6/101\$26.00 (C2010 IEEE

[9] Y.K. Cho , B. H. Park "Loop Stability Compensation Technique for Continuous-Time Common-Mode Feedback Circuits” 978-1-4673-9308-9/15/\$31.00 @2015 IEEE

[10] R. Ritter, M. Lorenz, Ch. Chu , M. Ortmanns “A High Open Loop Gain Common Mode Feedback Technique for Fully Differential Amplifiers” 978-1-4799-4885-7/14/\$31.00 @2014 IEEE

[11] X. Liu, J. F. McDonald ,L. Senior , "Design of Single-Stage Folded-Cascode Gain Boost Amplifier for 14bit 12.5Ms/S Pipelined Analog-to-Digital Converter” 978-1-4673-23963/12/\$31.00 (C)2012 IEEE 